\title{
The Analysis and Optimization of the VANET Topology
}

\author{
Yatie Xiao ${ }^{1, a}$, Luqun $\mathrm{Li}^{2, b}$ \\ 1 No. 100, Guilin Road, Shanghai, Xuhui District, Shanghai Normal University China \\ 2 No. 100, Guilin Road, Shanghai, Xuhui District, Shanghai Normal University China \\ agayani@163.com, ${ }^{b}$ liluqun@gmail.com
}

Keyword: VANET, Topology, Optimization, Adaptive

Abstract: Vehicular Ad-hoc Networks (VANET) is the research focus of ad-hoc network technology in recent years. Due to fast-moving and other properties of Vehicles, it is extremely easy to change the Topology of the VANET, and communication channels of VANET may become unstable. Then the system performance of the VANET drop overall. Based on the VANET Topology features, this paper proposes an adaptive VANAET Topology optimized Method .According to it, all the region of the VANET is divided into several logical spaces adaptively. In logical region, the method make the connection of V2V or V2I more efficient as possible. and between logical regions, it select several key nodes as the center nodes connecting with other logical regions. So it not only can reduce the average Degree distribution $D_{a}$ in and between logical regions but also improve the connection efficiency $\eta$ of the entire region. Thereby, providing professional traffic advices and emergency communication services for solving traffic congestion.

\section{Introduction}

VANET is a large and dynamic structure communication system consisting of Vehicles and Roadside infrastructures. Which is modeled on Wireless sensor Network (WSN), it makes fast-moving vehicles and stable Roadside Infrastructures as communication nodes, and uses the IEEE $802.11 \mathrm{p} / 1609$ communication protocol released in 2010 to provide timely and reliable Vehicle to Vehicle (V2V) and Vehicle to Infrastructure (V2I) communication service, Obviously the VANET is a gigantic dynamic communications system. The VANET schematic diagram is shown in Fig.1. Fig. 1 shows that many fast-moving vehicles and roadside infrastructures connect to others using protocol, which consist of the VANET.

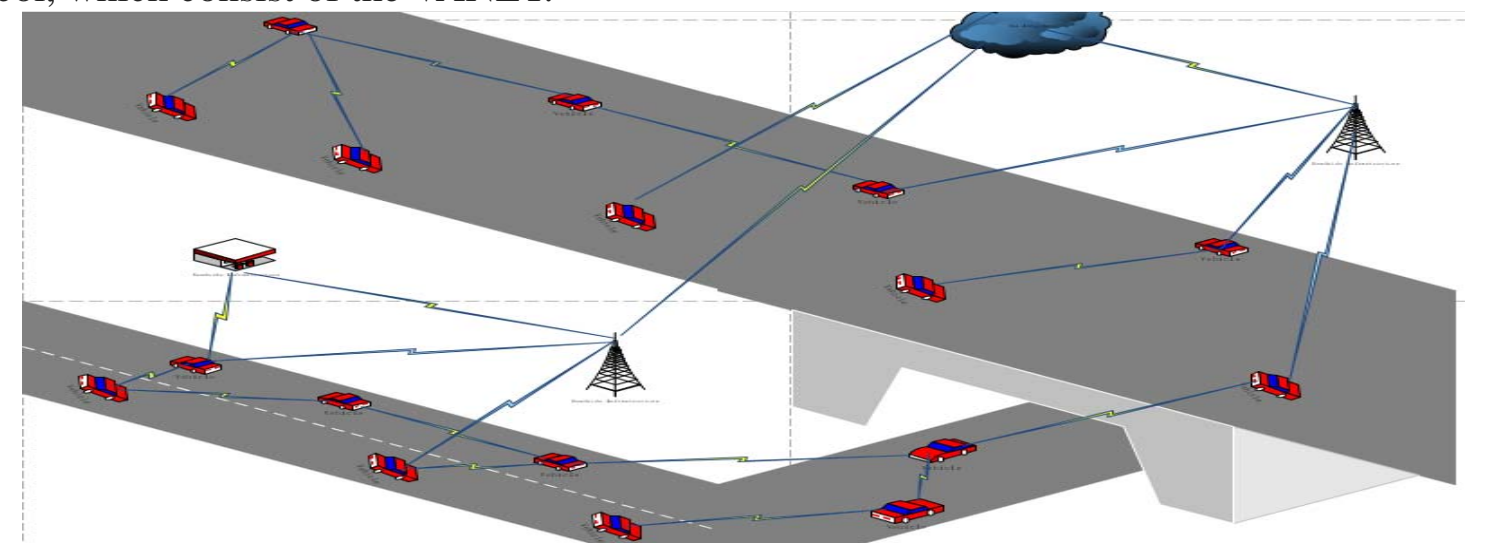

Fig. 1 The VANET Schematic diagram

In general, the robust topology is a prerequisite to ensure efficient communication performance of a communication system, due to fast-moving characteristics of vehicles in the VANET, It makes topology established by communicate processing can easily be changed, once a topology established is changed, communication channel of the VANET appropriate must be changed, system performance will be affected for sure. In the VANET, communication nodes, establishing communication channels and communication distance are essential elements of communication system topology analysis. Describing the topology of VANET accurately is to ensure the 
prerequisites for topology optimization. Based on the actual situation, this paper in the context of reasonable and accurate describe the topology of the VANET, combining the characteristics of the VANET, propose an adaptive method which makes the ideology of dividing sub-regions into topology optimization process, it can reduce the congestion of communications channels in theory, so as to improve the overall system of V2V and V2I communications efficiency.

\section{VANET Topology Definition}

VANET system is made up of vehicles and roadside infrastructures, the hardware components of the system are communication nodes, the degree of interaction between communication nodes determine the complexity of topology of the VANET, then topology complexity is an important aspect for communication system performance. In general, we use the graph ideology to describe the topology of the VANET. G (V, E) describe the whole communication system node and the node connection state. However communication nodes in the VANET are Vehicles and roadside infrastructures, only G (V, E) description for VANET topology is not comprehensive and enough. Taking the actual situation into account, we must add the signal transfer radius $\mathrm{R}$ of vehicles and the infrastructures to the topology description , so we define $G(V, E, R)$ as the VANET topology representation method $\mathrm{R}$ is expressed as maximum radius of signal transmission of communication nodes (Vehicles or roadside Infrastructures). For any communication node $V_{i}$ in the VANET, Its description contains V2V or V2I connection and maximum open signal transmission radius $\mathrm{R}_{\mathrm{i}}$. So the entire VANET topology can be described by the standard adjacency matrix A. Which is shown in Table.1.

Table.1 shows that adjacency matrix of the VANET topology, including communication nodes, connection status and signal transmission radius $\mathrm{R}_{\mathrm{i}}$.

$$
\mathrm{A}=\left[\begin{array}{ccccccccc} 
& R & V_{1} & V_{2} & V_{3} & V_{4} & V_{5} & \ldots & V_{i} \\
R & 0 & R_{1} & R_{2} & R_{3} & R_{4} & R_{5} & \ldots & R_{i} \\
V_{1} & R_{1} & 0 & 1 & 0 & 1 & 1 & \ldots & 0 \\
V_{2} & R_{2} & 1 & 0 & 0 & 0 & 1 & \ldots & 1 \\
V_{3} & R_{3} & 0 & 1 & 0 & 1 & 0 & \ldots & 1 \\
V_{4} & R_{4} & 0 & 1 & 0 & 0 & 1 & \ldots & 0 \\
V_{5} & R_{5} & 0 & 1 & 1 & 1 & 0 & \ldots & 0 \\
\ldots & \ldots & \ldots & \ldots & \ldots & \ldots & \ldots & 0 & \ldots \\
V_{i} & R_{i} & 0 & 1 & 1 & 1 & 0 & \ldots & 0
\end{array}\right]
$$

Table. 1 The adjacency matrix of the VANET Topology

\section{VANET Topology Analysis}

VANET communication system consist of vehicles and roadside infrastructures with full functions in a region and can perform free data transmission. By definition, we set an area within $\mathrm{M}^{*} \mathrm{M}$ $\left(\mathrm{Km}^{2}\right)$, its vehicles and roadside infrastructures maximum radius of signal transmission is $\mathrm{R}_{\mathrm{i}}$, the overall connection number of V2V and V2I is $\mathrm{N}$. In general, for a communication node $\mathrm{V}_{\mathrm{i}}$, its degree distribution $D_{i}$ is expressed as the number of connection with other nodes $D_{i}=\sum_{\substack{\mathrm{i} !=\mathrm{j} \\ \mathrm{j}=1}}^{N} \mathrm{a}_{i j}$. In this equation, $\mathrm{a}_{i j}$ is expressed as connection status that node $\mathrm{V}_{\mathrm{i}}$ to node $\mathrm{V}_{j}$ (1 means connected and 0 
indicates disconnected). Ideally, VANET average degree distribution $D_{a}=\frac{\sum_{\mathrm{i}=1}^{N} D_{i}}{N}$. By definition, we can see, when the value of $D_{a}$ is larger, the connection between nodes of VANET is more complex. We build a VANET nodes maximum connection status graph, which is shown in Figure.2.

Figure.2 shows that all nodes in VANET build their connections with others fully .

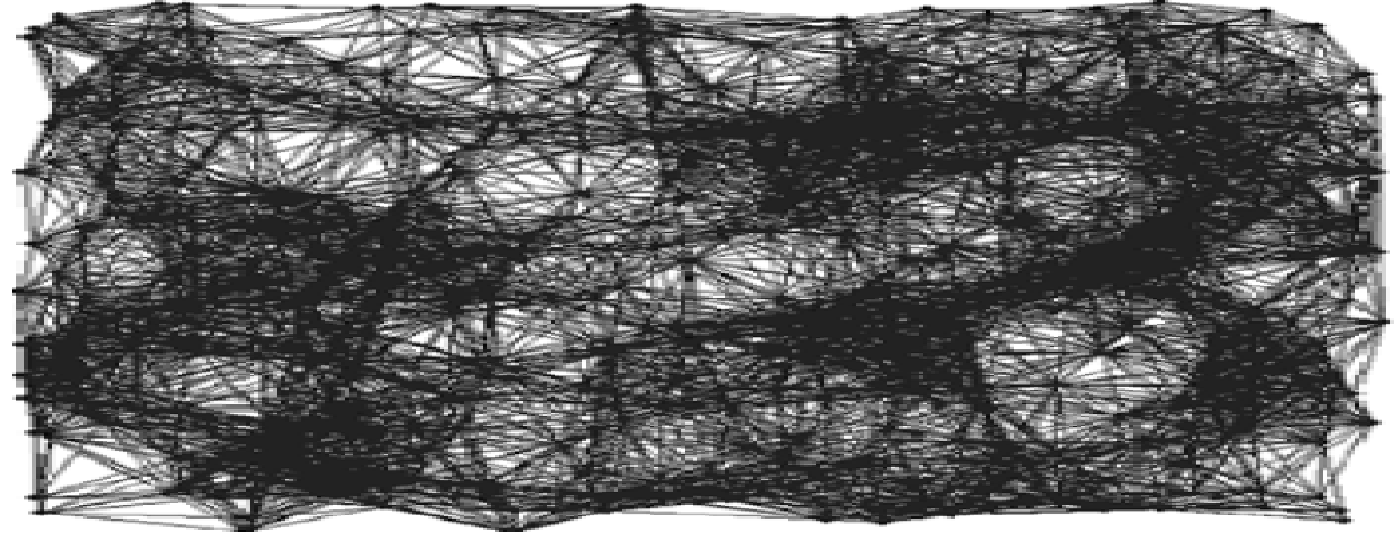

Figure.2 the maximum connections between nodes in the VANET

From the equation $D_{a}=\frac{\sum_{\mathrm{i}=1}^{N} D_{i}}{N}$, it is known that the value of $D_{a}$ is related to N. Make $D_{a} \approx$ $\mathrm{P}(\mathrm{N}) \quad(0<\mathrm{P}<1, \mathrm{P}$ is a probability parameter). We know that when $\mathrm{N}$ becomes extremely large, the probability distribution function $\mathrm{P}\left(D_{a}\right)$ approximately follows Poisson distribution with parameter $\lambda(0<\lambda<1)$, which is $\mathrm{P}\left(D_{a}\right) \approx \frac{e^{-\lambda} \lambda^{\mathrm{N}}}{\mathrm{N} !}$. Under ideal conditions, any node $\mathrm{V}_{\mathrm{i}}$ can connect with its neighbor nodes that distance between nodes is less than $R_{i}$, from which we can receive that $D_{\text {a max }}$ $=\frac{N \times(\mathrm{N}-1)}{2}$, in this case, any nodes can communicate with each other in VANET. Similarly, under another extreme conditions, each node can only connect to the rest of non-repetition neighbor nodes, at this time $\mathrm{D}_{\mathrm{a} \text { min }}=\mathrm{N}-1$. So based on above, $D_{a}$ can be obtained that:

$$
\mathrm{N}-1 \leq D_{a} \leq \frac{N \times(\mathrm{N}-1)}{2}
$$

\section{VANET Topology optimization}

Suppose the extreme condition, communication nodes in the VANET are valid at any time, when $D_{a}$ is equal to $\mathrm{N}-1$, any node can send data to neighbor destination nodes. However, in the actual situation, for the topology of the VANET in a region, it will not be so ideal. We still assume that the area of a region is $\mathrm{M}^{*} \mathrm{M}\left(\mathrm{Km}^{2}\right)$, its vehicles and roadside infrastructures signal transfer radius is $\mathrm{R}$, vehicles and the overall connection number of V2V and V2I is N. Owing to randomly distribution of vehicles in the VANET, we introduce an adaptive K-means clustering algorithm to partition all the communication nodes in the region into $\mathrm{K}$ logical spaces, the nodes in logical spaces can realize communication freely with neighbor nodes; besides it selects several nodes which are on the behave of logical spaces to achieve communication between other adjacent spaces. The optimized topology are shown in Figure.3.

Figure. 3 shows that after the processing of optimization, K logical spaces have been created and in space the connection status of nodes. 


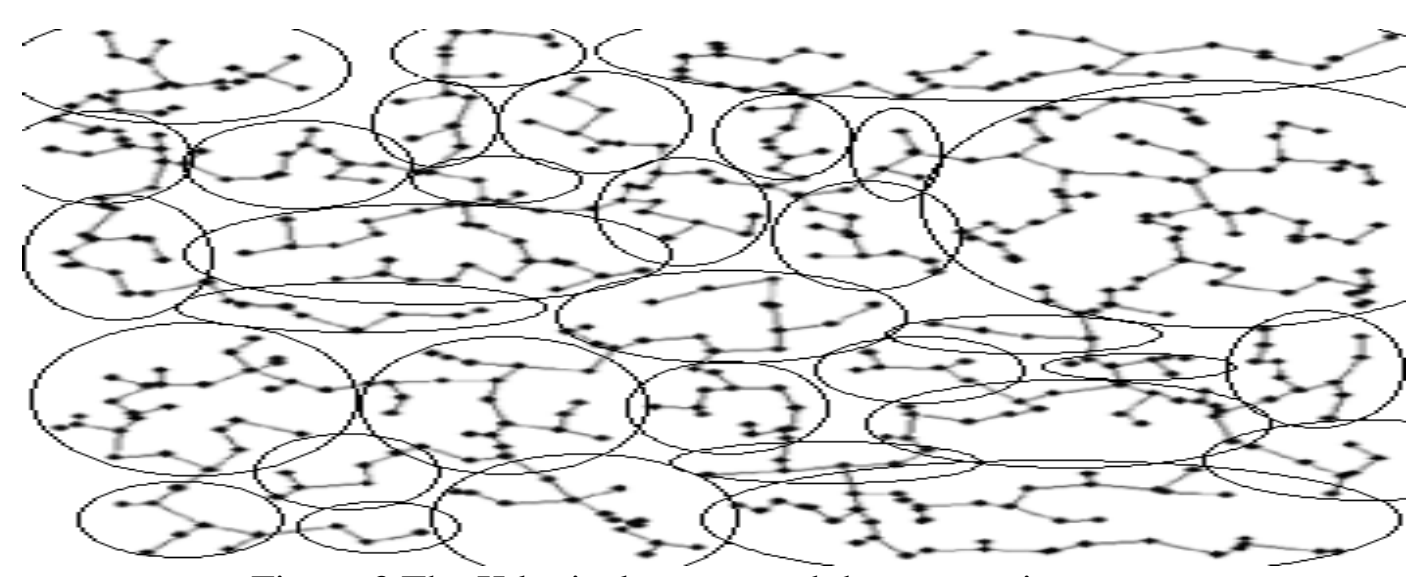

Figure.3 The K logical spaces and the connection status

It must be noted, Dividing the $\mathrm{M} * \mathrm{M}\left(\mathrm{Km}^{2}\right)$ region into $\mathrm{K}$ logical spaces is based on the condition that nodes in logical space are able to communicate with each other, namely: $B_{i j}=\left\{\mid b_{i}-\right.$ $\mathrm{b}_{\mathrm{j}}|<\mathrm{R},| \mathrm{b}_{\mathrm{i}}-\mathrm{b}_{\mathrm{j}} \mid$ shows the communication distance between node $\mathrm{i}$ and node $\left.\mathrm{j}\right\}$ holds. After optimized processing, we can receive that:

I , In logical region spaces $\mathrm{i}, \mathrm{D}_{\mathrm{ia}}=\sum_{\mathrm{i}=1}^{n_{i}} D_{i}, n_{i}$ is the number of communication nodes in logic spaces, so $D_{\text {iamax }}=\frac{n_{i} \times\left(n_{i}-1\right)}{2}$, and $D_{\text {iamin }}=n_{i}-1$;

II , Between logical spaces $\mathrm{j}, \mathrm{D}_{\mathrm{ja}}=\sum_{j=1}^{K} D_{j}, \mathrm{~K}$ is the number of the logic spaces. According to above method, we can get $D_{j a \max }=\frac{k \times(\mathrm{k}-1)}{2}$, and $D_{j a \min }=\mathrm{K}-1$.

So in all of the region, we can get:

$$
\begin{aligned}
& D_{a \max }=\sum_{i=1}^{k} \frac{n_{i} \times\left(n_{i}-1\right)}{2}+\frac{k \times(\mathrm{k}-1)}{2} \\
& D_{a \min }=\sum_{i=1}^{k}\left(n_{i}-1\right)+\mathrm{k}-1=\mathrm{N}-1
\end{aligned}
$$

According to above, it can be obtained that $D_{a}$ of the topology after optimized processing follows:

$$
\mathrm{N}-1 \leq D_{a} \leq \sum_{i=1}^{k} \frac{n_{i} \times\left(n_{i}-1\right)}{2}+\frac{k \times(\mathrm{k}-1)}{2}
$$

Obviously, the efficiency $\eta$ after the optimized processing can reach

$$
\eta=\frac{N^{2}-N-\left\{\left(\sum_{i=1}^{k} n_{i}^{2}-n_{i}\right)+k^{2}-\mathrm{k}\right\}}{N^{2}-N} \times 100 \%
$$

\section{Conclusion}

The complexity of the VANET topology directly relates to communication efficiency, we use a reasonably accurate representation of $\mathrm{G}(\mathrm{V}, \mathrm{E}, \mathrm{R})$ to describe the VANET topology, and introduce $D_{a}$ (average degree distribution) to quantify complex degree of the VANET topology, by using the adaptive K-means algorithm, it can simplify the complexity of the topology, improve V2V and V2I communication efficiency.

The next, we will learn more partitioning algorithms, and try to optimize the proposed algorithm, improving the efficiency of algorithm in partitioning node processing, while taking the location 
information of the vehicles or infrastructures and the influence of vehicles join and leaving the VANET into consideration in the VANET topology optimization process.

\section{References}

[1] Mohammad S A, Rasheed A, Qayyum A. VANET Architectures and Protocol Stacks: A Survey[C]// International Conference on Communication Technologies for Vehicles. Springer-Verlag, 2011:95-105.

[2] Broch, J., Johnson, D., Maltz, D.: The Dynamic Source Routing Protocol for Multi-Hop Wireless Ad Hoc Networks. Addison-Wesley, Reading (2001)

[3] Labrador M A, Wightman P M. Topology Control in Wireless Sensor Networks[M]. 2009.

[4] Bhatia S K. Adaptive K-Means Clustering.[C]// Seventeenth International Florida Artificial Intelligence Research Society Conference, Miami Beach, Florida, Usa. 2004.

[5] Akhtar N, Ozkasap O, Ergen S C. VANET topology characteristics under realistic mobility and channel models[J]. 2013:1774-1779.

[6] Mohammad S A, Rasheed A, Qayyum A. VANET Architectures and Protocol Stacks: A Survey[C]// International Conference on Communication Technologies for Vehicles. Springer-Verlag, 2011:95-105. 